\title{
Aspectos epidemiológicos da dengue nos biomas do Estado de Mato Grosso
}

Epidemiological aspects of dengue in biomes of Mato Grosso

Aspectos epidemiologicos de dengue en biomas del Mato Grosso

Evellyn Crystine Pessoa de Lima

Enfermeira pós graduanda, UNAMA, Brasil. evellyn_cristyne@hotmail.com

\section{Poliana Roma Greve Nodari}

Enfermeira mestranda, UNEMAT, Brasil. polianaroma@unemat.br

\section{Stephanie Sommerfeld de Lara}

Enfermeira mestranda, UFMT, Brasil.

Stephanie_sommerfeld@hotmail.com 


\section{RESUMO}

A dengue é uma doença infecciosa, considerada um problema de saúde pública, principalmente em países tropicais cujas condições são favoráveis a proliferação do vetor Aedes Aegypti. O escopo deste estudo foi identificar a taxa média de incidência dos biomas que integram o estado de Mato de Grosso e determinar qual bioma apresenta a maior média de incidência. Os dados foram obtidos pelo Departamento de Informática do Sistema Único de Saúde (DATASUS), considerando todos os casos de dengue registrados no estado nos anos de 2008 a 2012. Formulou-se a taxa média de incidência dos biomas que constatou que a maior incidência pertence ao Bioma Amazônico, seguido do bioma Cerrado e posteriormente o bioma Pantanal. No entanto, a taxa média poderia ser maior uma vez que ainda existam limitações dos vários sistemas de informações e subnotificações de casos, somados a não cobertura total da população pela Atenção primária no estado. A integração dos bancos de dados e uma maior abrangência dos serviços de saúde permitiria melhorar a vigilância deste agravo tornando-se uma alternativa útil para identificar os locais de alerta onde devem ser subsidiadas ações de promoção e proteção à população.

PALAVRA-CHAVE: Dengue, Saúde coletiva, ambiente.

\section{ABSTRACT}

Dengue is an infectious disease, considered a public health problem, especially in tropical countries where conditions are favorable for the proliferation of the vector Aedes aegypti. The scope of this study was to identify the average incidence rate of dengue in biomes that are part of the State of Mato Grosso and determine what biome has the highest average incidence. The data were obtained from the Department of the Unified Health System (DATASUS), taking into account all dengue cases reported in the state in the years 2008 to 2012. This body formulated the average incidence rate of biomes found that the highest incidence it belongs the Amazon biome, followed by the Cerrado and Pantanal later. However, the average rate could be higher, since there are still limitations of the various information systems and underreporting of cases, besides not having full coverage of primary care population in the state. The integration of databases and a wider range of health services could improve control, so it is useful for detecting the places warning which should subsidize the promotion and protection of the population alternative.

KEYWORDS: Dengue, Public health, environment.

\section{RESUMEN}

El dengue es una enfermedad infecciosa, considerada un problema de salud pública, especialmente en los países tropicales donde las condiciones son favorables para la proliferación del vector Aedes aegypti. El alcance de este estudio fue identificar la tasa media de incidencia del dengue en los biomas que forman parte del Estado de Mato Grosso y determinar qué bioma tiene la incidencia media más alta. Los datos se obtuvieron del Departamento del Sistema Único de Salud (DATASUS), teniendo en cuenta todos los casos de dengue notificados en el estado en los años 2008 a 2012.Este organismo formuló la tasa de incidencia media de biomas encontró que la incidencia más alta pertenece el bioma amazónico, seguido por el Cerrado y el Pantanal más tarde. Sin embargo, la tasa media podría ser mayor, ya que todavía hay limitaciones de los diversos sistemas de información y el subregistro de los casos, además de no tener una cobertura total de la población de atención primaria en el estado. La integración de bases de datos y una gama más amplia de servicios de salud podrían mejorar el control, por lo que es una alternativa útil para detectar los lugares de advertencia en el que se deben subvencionar la promoción y la protección a la población.

ALABRAS CLAVE: Dengue, la salud pública, el medio ambiente. 


\section{INTRODUÇÃO}

A dengue é uma doença infecciosa febril aguda, de curso benigno ou grave, dependendo de sua manifestação clínica, sendo considerado um problema de saúde pública, principalmente em países tropicais cujas condições são favoráveis a proliferação do vetor Aedes Aegypti (BRASIL, 2008). Em 2014 o Ministério da Saúde alterou a classificação da doença segundo recomendação da Organização Mundial da Saúde (OMS). Nessa nova classificação as formas de apresentação da doença passaram a se classificar como: Dengue, Dengue com sinais de alarme e Dengue grave (BRASIL, 2014).

A doença é transmitida pela picada do mosquito infectado (fêmeas). O período de incubação no homem pode variar de 3 a 15 dias, sendo, em média, de 5 a 6 dias. Sua manifestação clinica ocorre de forma variada, desde infecções a formas hemorrágicas graves. São considerados casos suspeitos de dengue, paciente que tenha doença febril aguda, com duração máxima de 7 dias, acompanhada de pelo menos dois dos seguintes sintomas: cefaleia, dor retro orbital, mialgia, artralgia, prostração, exantema que deve ter estado, nos últimos quinze dias, em área onde esteja ocorrendo transmissão de Dengue ou tenha a presença de Aedes aegypti (BRASIL, 2013).

As notificações de casos da dengue devem ocorrer em um prazo máximo de até 24 horas, a partir da suspeita inicial, as Secretarias Estaduais de Saúde e as Secretarias Municipais de Saúde, também deverão informar imediatamente à Secretaria de Vigilância em Saúde (SVS). É importante ressaltar que a notificação compulsória é obrigatória a todos os profissionais de saúde e outros no exercício da profissão, bem como os responsáveis por organizações e estabelecimentos públicos e particulares de saúde e de ensino, em conformidade com a Lei no 6.259 (BRASIL, 2011).

Segundo a Organização Panamericana De Saúde (2011), foram notificados em 2011, 979.774 casos de dengue, nas áreas tropicais e subtropicais das Américas. No Brasil, foram registrados 727.803 casos ao total no mesmo ano. Mato Grosso apresentou o segundo menor número de casos da região centro-oeste em 2011 com 5.170 casos de dengue (OPAS, 2016).

O mapeamento de doenças vem sendo utilizado na saúde pública como um mecanismo de orientação e controle de endemias, de forma a auxiliar e conduzir políticas públicas sobre os problemas ambientais e de saúde decorrentes de várias regiões (FERNANDES; NEVES; NEVES, 2014). Nesse sentido esse artigo buscar mapear por biomas, a situação epidemiológica da dengue no estado nos anos de $2008-2012$.

\section{OBJETIVO}

Identificar a taxa média de incidência de dengue nos biomas que integram o estado de Mato de Grosso e determinar qual bioma apresenta a maior média de incidência. 


\section{MÉTODOS}

\section{1 Área de Estudo}

O estado de Mato Grosso está localizado na região centro-oeste do Brasil. De acordo com Censo (2010) possui 3.035.122 habitantes distribuídas em uma área territorial de $903.357 \mathrm{~km}^{2}$, com índice de IDH de 0,725. Suas dimensões abrigam 141 municípios distribuídos em três tipos de biomas: amazônico, cerrado e pantanal que compõe seu território ambiental. A taxa de crescimento do Produto Interno Bruto (PIB) é de 1,6\% do PIB Brasil.

\subsection{Desenho do estudo}

O estudo tem caráter epidemiológico e descritivo, realizado a partir de dados obtidos através do Departamento de Informática do Sistema Único de Saúde (DATASUS). Foram considerados casos de dengues confirmados, registrados no estado, entre os anos de 2008 a 2012. Todos os casos notificados dos municípios da unidade federativa foram tabelados, depois separados de acordo com os biomas do qual pertencem. Após a tabulação, foram realizadas as taxas de incidência por cem mil habitantes de cada município, e em seguida as taxas referentes aos biomas. A população residente foi adquirida no sítio do Instituto Brasileiro de Geografia e Estatística (IBGE), censo 2010.

\subsection{Análise de dados}

Para a análise a nível municipal, foram realizadas taxas médias de incidência através da equação:

Taxa média de incidência

$=\frac{\text { Soma dos casos registrados no ano de } 2008 \text { a } 2012 / 5}{\text { População residente no município X }} \times 100.000$ Habitantes

Em relação à análise por biomas, a equação descrita a seguir:

$$
\begin{aligned}
& \text { Taxa média de incidência por bioma } \\
& \qquad=\frac{\text { Soma dos casos no ano de } 2008 \text { a 2012/5 }}{\text { População residente no bioma x }} \times 100.000 \text { Habitantes }
\end{aligned}
$$

Foram gerados mapas temáticos com a distribuição das taxas medias de incidência classificadas em quartis de igual frequência: baixo, médio, alto, e muito alto.

\section{RESULTADOS}

A dengue é uma das doenças mais frequentes no Brasil, atingindo todos os estados, sem distinção de classe social. Tem como principal vetor na América o Aedes aegypti, podendo também ser transmitido pelo Aedes albopictus, no entanto, ainda não há registros de casos por esse vetor no país. Possui como características cor escura, rajado de branco nas patas e no corpo. Sua transmissão ocorre pela picada da fêmea infectada (BRASIL, 2008).

O seu ciclo de vida apresenta quatro fases: ovo, larva, pupa e adulto. Nessa fase costumam viver em média entre 30 a 35 dias. 0 mosquito fêmea pode por de 4 a 6 seis vezes, ovos 
durante sua vida, sendo cerca de 100 ovos por vez. Importante ressaltar que os ovos podem sobreviver por até 450 dias mesmo que o lugar onde foi depositado fique seco, podendo ficar ativo novamente caso o lugar receber agua novamente (BRASIL, 2008).

Segundo Lana (2009), o controle da dinâmica populacional e dispersão do vetor é a forma mais viável de contenção da doença, uma vez que a vacina, ainda não esta acessível para população. Contudo se faz necessário o conhecimento dos mecanismos de propagação da doença, para criação e planejamento de programas para controle que possam ser implementadas.

De acordo com Tauil (2002) cerca de 2,5 bilhões de pessoas estão em risco de adquirirem dengue, principalmente em países tropicais onde à temperatura e a umidade favorece a proliferação do mosquito vetor. Ferreira e Yang (2003) estudaram os ciclos de vida do mosquito e evidenciaram que a temperatura do ambiente é determinante em relação à dinâmica da população do vetor uma vez que a variável climática controla o tempo de desenvolvimento do mosquito. Este estudo corrobora com o de Mendonça (2003) que afirma que o aumento da temperatura diminui o período de incubação do vírus, Lana (2009) reforça que com a diminuição do período de incubação do vírus, a transmissibilidade ocorre de forma mais rápido o que contribuiria para que uma epidemia ocorresse mais depressa. O A. Aegypti tem mostrado capacidade de adaptação em diferentes ambientes desfavoráveis como em altitudes elevadas e larvas em água poluída (TAUIL, 2002).

A sua proliferação tem múltiplos condicionantes; fluxo rural-urbano intenso uma vez que mais de $80 \%$ da população brasileira vive hoje em área urbana, e estas não conseguem oferecer condições satisfatórias de habitação e de saneamento básico forçando uma parte da população a viverem em lugares onde o abastecimento de água e a coleta de dejetos, são irregulares, o armazenamento de água para consumo; fator que favorece a proliferação do mosquito vetor, o processo industrial moderno e a não reciclagem; de embalagens descartáveis, contribui para a proliferação dos mosquitos, são um dos fatores que facilitam a disseminação da doença (TAUIL, 2002).

A não disponibilidade de serviços de saneamento ambiental em quantidade e qualidade adequadas, incluindo o abastecimento de água e coleta de lixo influencia na reprodução do vetor (MALAVASI, 2011). Nesse Contexto observa-se que a saúde de uma população esta intimamente ligada a fatores ambientais, sociais, políticos e econômicos na qual esta inserida podendo estes agir de forma positiva ou negativa sobre seu bem-estar.

O estado de Mato Grosso passou por um acelerado crescimento demográfico nas últimas décadas, em um período de 25 anos a população mato-grossense cresceu 4,5 vezes. A expansão demográfica fica mais visível quando analisados as taxas de urbanização de 1970 de $38,9 \%$ para $76,8 \%$ no ano de 2004 . No entanto alguns segmentos como o esgotamento sanitário, não acompanhou o aumento populacional uma vez que apenas 59,5\% dos domićlios detinham de saneamento básico (sendo $17 \%$ ligada à rede coletora e $42,3 \%$ de fossa séptica). A precariedade dos serviços também abrange o abastecimento de água sendo o estado da região centro-oeste com menor proporção de domicílios dotados com água encanada. A coleta de lixo também se revela um problema no estado que atende apenas $85 \%$ dos domicílios, um valor percentual abaixo da média regional e nacional (MATO GROSSO, 2004).

Em 2012, Mato Grosso ocupou o $4^{\circ}$ lugar no Ranking dos estados com maior número de casos de dengue (BRASIL, 2015). Segundo levantamento realizado pelo Conselho Federal de 
Medicina o estado ocupou o $16^{\circ}$ lugar no ranking de gasto público per capita em saúde no ano de 2013. Cada mato-grossense custou para os cofres públicos cerca de $\mathrm{R} \$ 316,08$ equivalente a $\mathrm{R} \$ \mathbf{0 , 8 8}$ por dia. Foi destinada a saúde da população cerca de $\mathrm{R} \$ 1$ bilhão de reais, no entanto apenas 34 milhões foram destinados a Atenção Básica.

Vale ressaltar que a Atenção Básica, as Estratégias de saúde da Família (ESF) exerce papel fundamental no diagnóstico da dengue que segundo o Ministério da Saúde pode ser realizada através de sorologia ou isolamento viral. Além do exame clínico as (ESF) são imprescindíveis na coleta de informações sobre a doença e dos dados epidemiológicos, uma vez que a coleta de dados primária é essencial para qualquer sistema de informação visto que orientam políticas de saúde pública (BOCHNER et al., 2012).

Segundo Análise dos indicadores da Atenção Primaria de Mato Grosso (2011) o estado obtinha 586 ESF, cobrindo $67 \%$ da população mato-grossense. Apesar da Dengue não estar incluída como foco prioritário na Política de Atenção Básica (PNAB), seu potencial de disseminação é preocupante, sendo necessárias intervenções precoce de controle para reduzir resultados desfavoráveis.

Em Mato Grosso, foram contabilizados 133.280 casos de dengue no estado entre 2008-2012. Em 2009, houve o maior registro de casos de dengue (53.660), se comparado aos demais anos pesquisados. Contudo, em 2011 observou-se uma diminuição significativa dos casos de dengue, possivelmente decorrente das campanhas de combate ao mosquito. A média de casos de 2008-2012 foi de 26.656 por ano, com uma taxa de incidência de 878,3 casos por cem mil habitantes.

FIGURA 1- Taxa média de incidência de dengue por bioma na unidade federativa de Mato Grosso, 2008 a 2012.

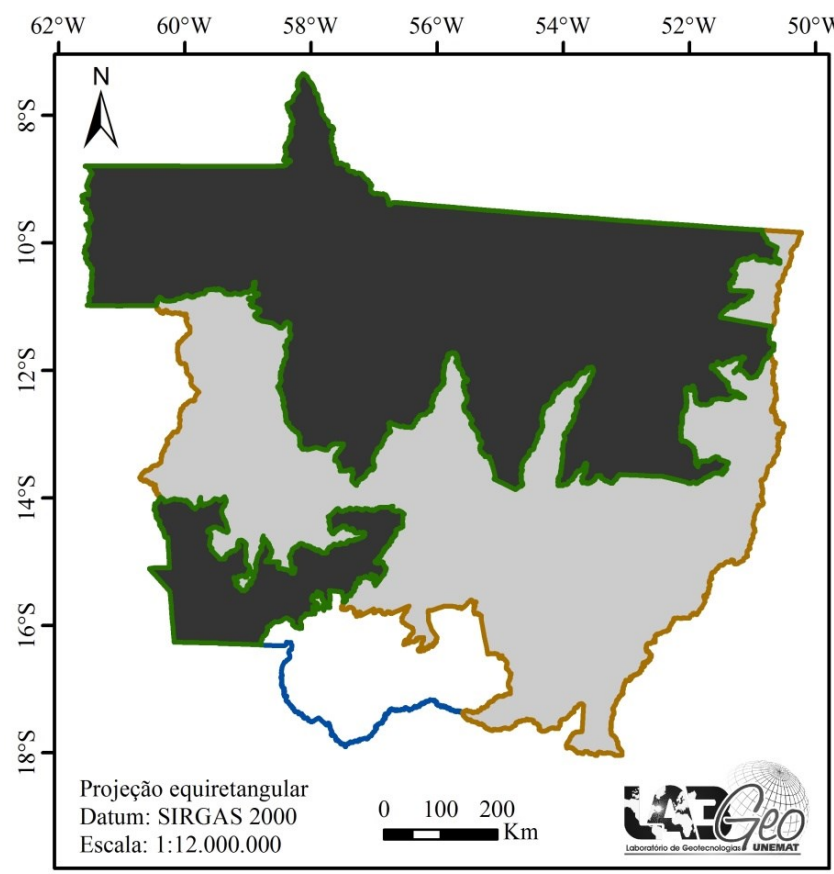

Legenda

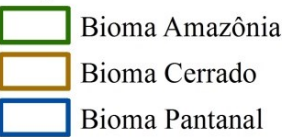

Taxa média de incidência por 100 mil habitantes

901,4 a 947 - Muito alto 855,7 a 901,3 - Alto 810 a 855,6 - Médio 764,3 a 809,9 - Baixo 
O bioma amazônico concentra-se no norte do estado, onde situa 74 municípios, o que corresponde a $35 \%$ da população do estado. Foram identificados nos últimos cinco anos 50.678 casos de dengue, com média anual de 10.135,6 casos. Em 2009, ocorreu a maior incidência de casos dos anos estudados, no entanto observou-se uma diminuição nos próximos dois anos seguintes, principalmente em 2011 onde se registrou o menor número de casos. A taxa de incidência da dengue no bioma amazônico é de 947,0 por cem mil habitantes.

O cerrado é o principal bioma do centro-oeste brasileiro, cobrindo 38,29\% de todo território de Mato Grosso, localizado principalmente nas depressões de Alto Paraguai - Guaporé, o sul e o sudeste do planalto dos Parecis e ao sul do paralelo 13으, até os limites de Mato Grosso do Sul (GROSSO et al., 2016). Abrange 65 municípios e abriga a parte mais populosa do estado cerca de $60 \%$ do total. Verificou-se que nos anos de 2008-2012, obteve-se um total de 81.119 casos, com uma média anual de 16223,8 casos com taxa de incidência de 846,5 por cem mil habitantes.

Dividido entre o Estado de Mato Grosso e o Mato Grosso do Sul, o Pantanal é a representa um importante ecossistema para o equilíbrio ecológico e para a sustentabilidade ambiental do planeta. O solo pantaneiro não é adequado à agricultura por ser considerado de baixa fertilidade em áreas úmidas. Sua atividade econômica em maior evidência na região é a agropecuária (GROSSO, 2013).

O bioma Pantanal é a maior área alagável do planeta, mas ocupa apenas 7,2 \% do estado, sendo considerado Patrimônio Natural mundial e Reserva da Biosfera pela UNESCO (GROSSO et al., 2016). Abriga apenas $5 \%$ da população do estado, distribuídos em 05 municípios. Nos de 2008 a 2012 foram notificados 5.757 casos de dengue, uma média de 1.151,4 casos por ano com uma taxa média de incidência de 764,3.

Figura 2 - Taxa média de incidência de dengue por município na unidade federativa de Mato Grosso, 2008 a 2012.

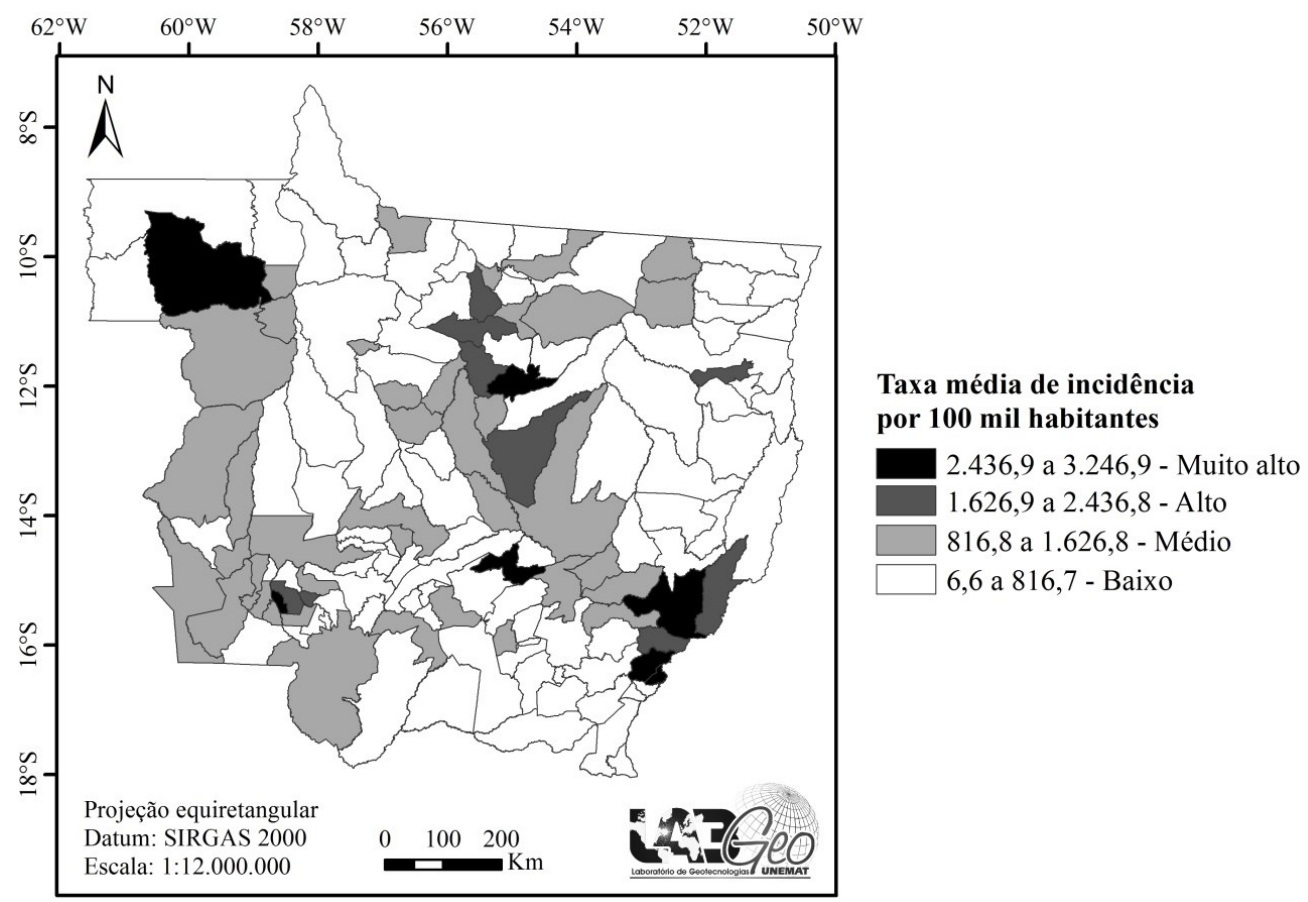


Os maiores índices de casos da dengue pertencem aos municípios de Ribeirãozinho, Nova Brasilândia e Torixoréu que estão nos três primeiros lugares no estado e integram o bioma Cerrado. Seguidos dos municípios de Indiavaí e Aripuanã que apresentam as taxas mais altas do bioma Amazônico, sendo a $4^{\circ}$ e $5^{\circ}$ maior taxa do estado. A Cidade de Cáceres foi o município que apresentou a taxa de incidência mais alta do bioma Pantanal com 1018,6 sendo maior que a taxa de incidência do bioma que foi de 764,3 e ocupa o $43^{\circ}$ lugar do estado. É importante ressaltar que são as intervenções humanas sobre o meio ambiente, que favorecem organismos portadores de doenças, como o Aedes, se estabelecerem uma vez que este se prolifera em habitats artificialmente criados por humanos.

\section{CONCLUSÃO}

Coma realização desta pesquisa epidemiológica podemos concluir que o bioma com a taxa média de incidência mais alta foi o bioma Amazônico com a média de 947,0 casos por cem mil habitantes, seguido do bioma Cerrado com média de 846,5 casos de dengue e posteriormente o bioma Pantanal com 764,3 de incidência média nos anos de 2008 a 2012. No entanto, a taxa média poderia ser maior uma vez que ainda existam limitações dos vários sistemas de informações e subnotificações de casos, somados a não cobertura total da população pela Atenção primária no estado. A integração dos bancos de dados e uma maior abrangência dos serviços de saúde permitiria melhorar a vigilância deste agravo tornando-se uma alternativa útil para identificar os locais de alerta onde devem ser subsidiadas ações de promoção e proteção à população.

\section{REFERÊNCIAS}

BOCHNER, R. et al. Qualidade da informação: a importância do dado primário, o princípio de tudo. Tendências da Pesquisa Brasileira em Ciência da Informação, v. 4, p. 3526-3538, 2012.

BRASIL. Orientações para Ações de Vigilância Epidemiológica. Journal of Chemical Information and Modeling. [S.I: s.n.], 2013. v. 53. p. 1689-1699.

BRASIL. Plano de Acao para a Prevencao e Controle do Desmatamento na Amazonia Legal. p. 156, 2004.

BRASIL. Portaria N 104, de 25 de Janeiro de 2011. PhD Proposal, v. 1, 2011.

BRASIL. Superintendência de vigilância em saúde. p. 1-14, 2014.

BRASIL. Vigilância em Saúde: Dengue, Esquistossomose, Hanseníase, Malária, Tracoma e Tuberculose. Cadernos de Atenção Básica, n. 21, p. 195, 2008.

BRASIL, S. Casos de Dengue. Brasil, Regiões e Unidades Federadas. p. 2015, 2015. Disponível em: $\quad<$ http://portalsaude.saude.gov.br/index.php/o-ministerio/principal/leia-mais-oministerio/752-secretaria-svs/vigilancia-de-a-a-z/raiva/11431-situacao-epidemiologica-dados>.

CONSELHO FEDERAL DE MEDICINA-CFM.Disponível em: http://portal.cfm.org.br/>acesso em: 07 abril.2016 
ESTADO DE MATO GROSSO. Plano de desenvolvimento do estado de Mato Grosso. [S.I: s.n.], 2013. v. 53.

FERNANDES, R.; NEVES, S.; NEVES, R. Disponível em: http://www.redalyc.org/articulo.oa?id=53423372010. Boletim Goiano de Geografia, v. 34, n. n 1, p. 93-110, 2014.

Ficha Catalográfica Brasil. Mato Grosso. Secretaria de Estado de Saúde. Superintendência de Atenção à Saúde. Coordenadoria de Atenção Primária. Análise dos Indicadores de Atenção Primária - Sistema de Informação da Atenção Básica: modelo de atenção Estratégia Saúde da Familia.2009.Secretaria de Estado de Saúde de Mato Grosso - Cuiabá, 2011.37 páginas.

GROSSO, M. et al. Aspectos Geográficos do Estado de Mato Grosso. p. 2-4, 2016.

LANA, R. M. Dissertação de Mestrado : “ Modelos Dinâmicos Acoplados para Simulação da Ecologia do vetor Aedes aegypti ". Ecologia, 2009.

MALAVASI, H. G. Análise Espacial Da Epidemia De Dengue Em Campinas/ Sp. p. 117, 2011.

MENDONÇA, F. Aquecimento global e saúde: uma perspectiva geográfica - notas introdutórias. Terra Livre v.1(20), p.205-221,2003.

MINISTÉRIO DO MEIO AMBIENTE. Plano de Ação para Prevenção e Controle do Desmatamento e das Queimadas -Cerrado. Brasília: [s.n.], 2011.

ORGANIZAÇÃO PANAMERICANA DE SAÚDE. Alerta Epidemiológica: Dengue en las Américas. p. 1-3, 2011.

TAUIL, P. L. Critical aspects of dengue control in Brazil. Cadernos de Saúde Pública, v. 18, n. 3, p. 867-871, 2002.

YANG, H.M., FERREIRA, C.P., TERNES, S. Dinâmica Populacional do Vetor Transmissor da Dengue. v.02 p.287-296,2003. 